\title{
NI LabVIEW Based Camera System Used for Gait Detection
}

\author{
Dániel Salánki \\ University of Debrecen \\ Faculty of Engineering Mechatronics \\ Debrecen, Hungary \\ danisalanki@yahoo.com
}

\author{
Kornél Sarvajcz \\ University of Debrecen \\ Faculty of Engineering Mechatronics \\ Debrecen, Hungary \\ sarvajcz@eng.unideb.hu
}

\begin{abstract}
In these times, with the development of the world, biometric identification systems are becoming more and more widespread. Access control systems, but even the most mid-range smartphones have biometric authentication features, and even ID cards can include a person's fingerprint. The research group previously realized a rudimentary gait recognition system, which was upgraded to a multicamera system with high-resolution cameras and instead of reference points, the new version recognizes different templates. The program can compare and evaluate the functions that are matched to the reference curve and the current curve in a specific way, whether two walking images are identical. The comparison is decided by the definite integrals of the two suited functions. The self-developed gait recognition system was tested by the research team on several test subjects and according to the results, permission was never given to a strange person.
\end{abstract}

Keywords - camera system; gait image; identification; integral calculus; evaluation.

\section{INTRODUCTION}

There are several disciplines where motion tracking and motion capture devices play an important role. Examples include automation, robotics and computer animation. Different application areas may have different specific requirements, other needs may be important. There are places where it is enough to follow one or two independent points, elsewhere, e.g. at character animation, the following points form a complex transformation hierarchy where the complete rigid transformation of each element is required [1].

Compared to physiological biometric identification (fingerprint identification, iris and face identification, ear tag geometry identification), walking has the advantage of being able to identify human subjects with low-resolution or hidden camera images [2]. Physiological biometric methods are incapable of reliably recognizing non-cooperating individuals, especially at a distance, under changing environmental conditions in the real world [3]. However, the accuracy of gait recognition can be influenced by several factors, such as the angle of view, which changes the matching of visual characteristics with each other [4].

\section{The CREated Gait ReCognition Software [5]}

In 2017, the research team set up an initial walk detection system with a $640 \times 480$ resolution camera in LabVIEW [6]. The improved version will be presented below.

The main change of the newer version compared to the previous one is that the program can now handle two cameras instead of one, and instead of real time processing due to the higher system requirements, the walking is recorded, then the video file is analysed by the program. The cameras were placed side by side to complement each other. A further modification compared to the old version is that instead of point recognition, pattern recognition is done, which can better filter out the false sensed points, and that instead of comparing the coefficients, the definite integral values of the functions are compared.

The front panel of the new version of the program is shown in Fig. 1. The user can switch between 4 tabs using Tab Control. The first tab shows the recordings made by the two cameras. Under the displays can be selected the computer location of the video files. There is a larger green lamp that flashes when the test subject is recognized. There are 8 smaller lamps above the larger one, which indicate the logical values of 8 comparisons. In the bottom line, the locations of the templates can be selected, in this case 6,3 per camera, because in each case 3 templates were placed on the test subject: one on the ulna proximalis (forearm), one on the fibula proximalis (knee) and one on half distance between the right fibula proximalis and the outer ankle (calf). These templates will be searched in the videos. This version no longer requires a Stop button because the program stops automatically after analysing the videos.

On the second tab of the front panel, the coordinates of the detected templates are stored in arrays $\mathrm{X}$ and $\mathrm{Y}$, respectively. On the third tab, the resulting walking curves are displayed separately for the first and second video, and the curve described by the template on the forearm is evaluated for both videos. During the evaluation, the program fits a function on the curve described by the template and calculates the definite integral of it between 0 and 1920 . 


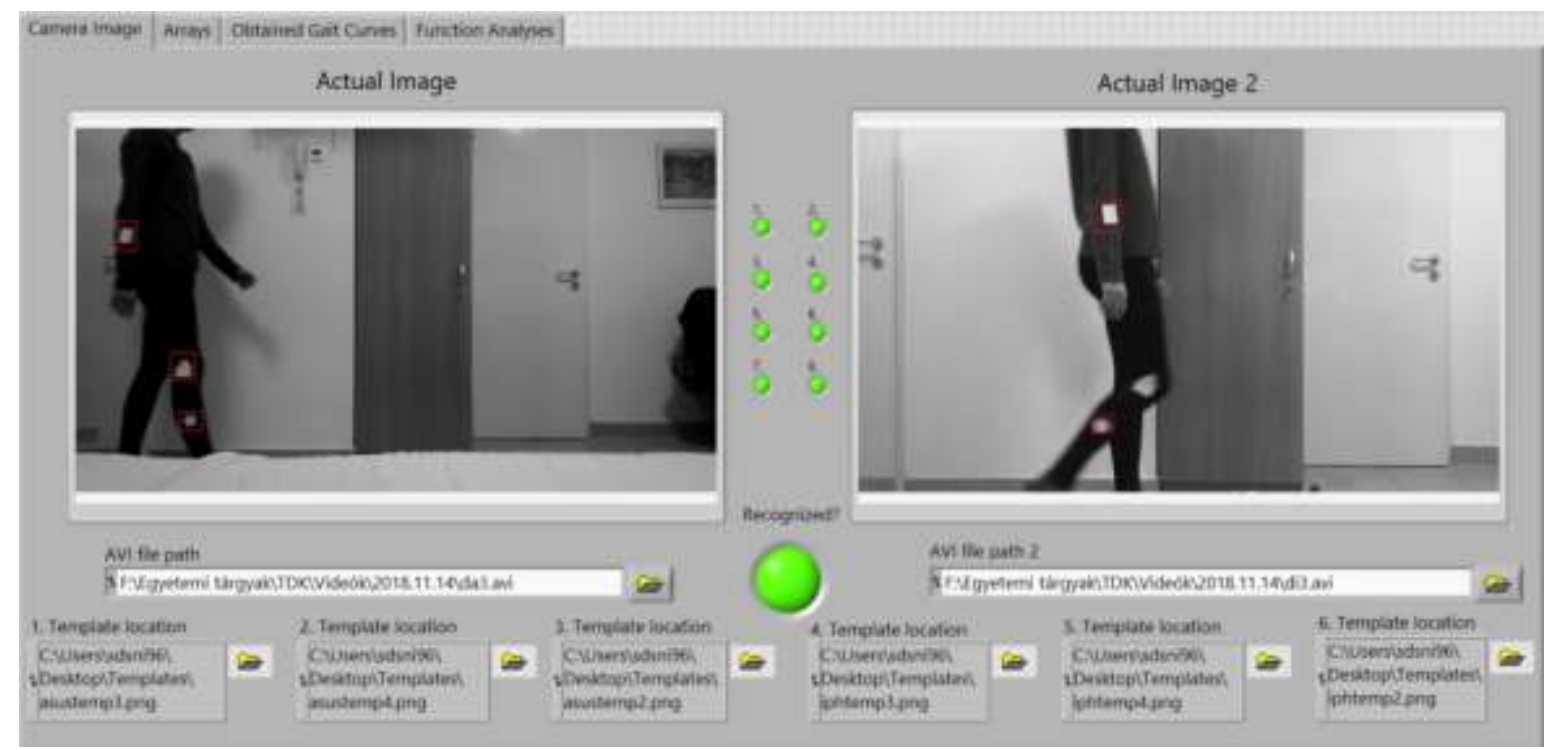

Fig. 1. The First Tab of the Front Panel of Gait Recognition Software.

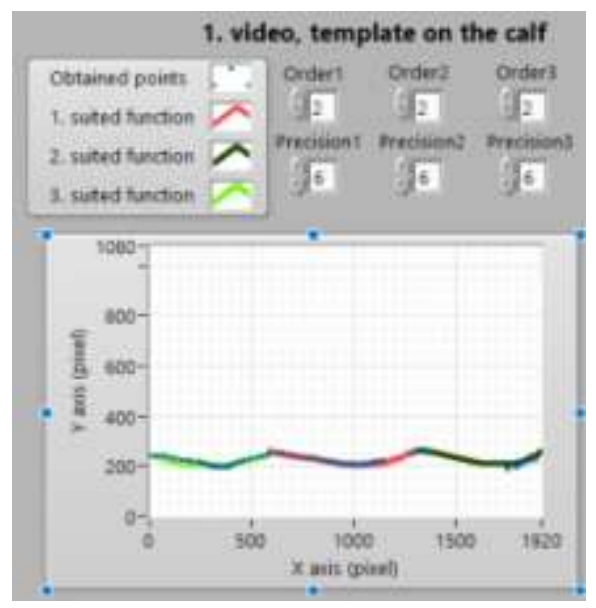

Fig. 2. A Part of the Fourth Tab.

The fourth tab contains the evaluation of the other walking curves. The second graph shows the curves drawn by the template on the calf in the first video, as shown in Fig. 2. The third and fourth graphs show the suited functions on the curves described by the knee's and the calf's template in the second video. For the forearm and knee-mounted templates, a second-degree alignment is performed, because these templates describe a path with a small curvature, so they can be considered straight with a good approximation, but because of better accuracy, the second-degree member was also considered. However, the calf template's curve cannot be written with a single function. Based on our observations, about 3 steps fit into the camera image, which can be described with 3 second-degree functions. The integration limits are the local maximums of the functions.

Taking the average of several 10 measurements, the limits of the definite integrals of each function have been determined. If the difference from this limit is within $5 \%$, or in some cases within $12.5 \%$, then a logical 1 is given from that part of the program. In the case of the calf template, it was formed the sum of the values of the definite integrals since a smaller standard deviation was observed between the individual values, but their sum was approximately constant during the measurements. The program makes a total of 8 comparisons. If each one closes with a logical 1, the green light will flash, and the test subject will be noted as recognized.

\section{RESULTS [5]}

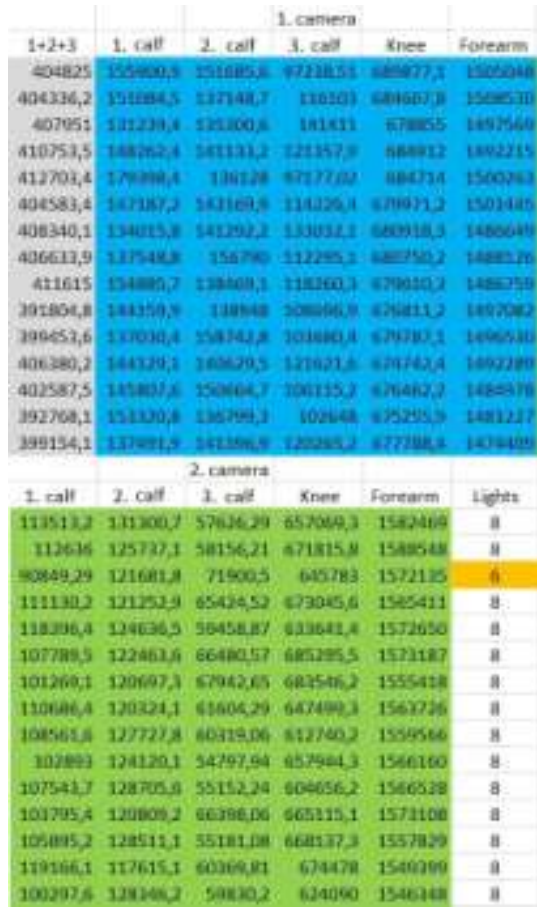

Fig. 3. Gait Data from a Known Test Subject.

After setting up the camera system, the research team tested it with several subjects. The measurements were made in normal clothing (trousers and long-sleeve T-Shirt). The test subjects took the first step with their left foot. Fig. 3 . 
illustrates the results of a test subject known by the program. The first grey column shows the sum of the values of the first 3 blue columns. The blue columns show the results of the first video. The values represent the definite integrals of the functions suited on the curves drawn by the templates, the first, second, and third functions of the template placed on the calf, the function of the knee, and the curve of the template fitted to the forearm, respectively. The green columns show data obtained from the video produced by the second camera in a similar division to the first one. The last column shows the number of flashing small lights on the user interface.

The program correctly detected the known test subject 14 times out of 15 (Fig. 3.). In this case, the false rejection rate (FRR) was $6.25 \%$ (correct recognition rate: $93.75 \%$ ). In the case of a similar test subject to the known one, but unknown to the system, the program has never given the permission, so the False Acceptance Rate (FAR) was $0 \%$.

\section{COMPARISON With OTHER GAIT RECOGNITION SYSTEMS}

Dacheng Tao, Xuelong Li, Xindong Wu, and Stephen J. Maybank have been tested a Gabor-based gait recognition system. Gabor filters can be used to amplify linear structures in a given direction. With this method, a detection rate of $92 \%$ was obtained from different views and in footwear, and $87 \%$ for test subjects with a briefcase [7].

The GEI-based (Gait Energy Image) system tested by Khalid Bashir, Tao Xiang, and Shaogang Gong has been run by multiple databases without the cooperation of test subjects. Based on the CASIASetA database, where there is no packet and no big jacket with test subjects, the recognition rate was $100 \%$, however, for CASIASetB and CASIASetC, where the test subjects are with pack or wearing large jackets, the detection rate was $77.8 \%$, and $43.1 \%$ [8].

Chin Poo Lee, Alan W.C. Tan and Shing Chiang Tan used the TBP - Transient Binary Patterns method to develop walking detection system and compared it with other methods. The TBP and GEI methods resulted in a 99\% detection rate. The Local Binary Patterns from Three Orthogonal Planes (LBP-TOP) resulted in only a $49 \%$ detection rate [9].

Bence Gálai and Csaba Benedek investigated districtbased personal identification and action recognition with LiDAR sensors (Light Detection and Ranging). Several people have been introduced to their systems at one time, with a $100 \%$ detection rate for up to 5 people, and then gradually reduced to $75 \%$ for 22 people after which it stagnates [10].

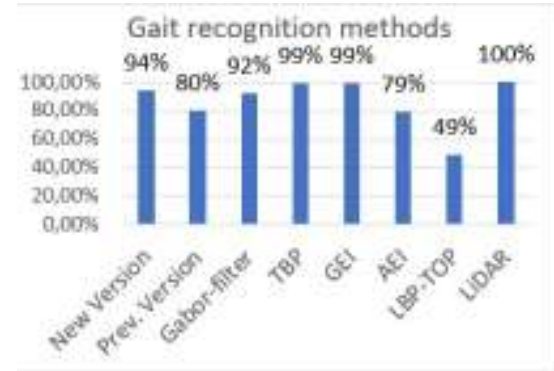

Fig. 4. The Research Team's Gait Recognition System Compared with Other Systems.
Fig. 4 summarizes the accuracy of the current and previous systems of the research group and other systems. Based on this, we can say that the most accurate gait detection systems are the LiDAR (100\%), TBP (99\%) and GEI (99\%). The system set up by the research team is also good with its $93.75 \%$ recognition rate, while the least efficient is the LBPTOP system with $49 \%$.

\section{CONCLUSION}

In summary, the research team has succeeded in creating a complex gait recognition system in NI LabVIEW environment that can detect multiple samples using multiple cameras and is able to fit predefined curve on the collected samples. The program is also able to compare the values of the reference curve and the current curve in a specific way and to evaluate if the image of the two curves is the same. The self-developed gait recognition system has been tested on several individuals, which never gave permission to a foreign person.

In the future, the processing speed of the program could be improved with a camera or a software that can be used to immediately save the video in AVI format. The 2014 LabVIEW version used in the research is only capable of postprocessing AVI videos. Writing the program in other software (e.g. OpenCV), which is capable of handling MP4 files, or in which a high-quality real-time camcorder does not hold back the performance, can also improve the processing speed.

\section{Acknowledgement}

The work/publication is supported by the EFOP-3.6.1-16$2016-00022$ project. The project is co-financed by the European Union and the European Social Fund.

\section{References}

[1] D. Viktor, „Optikai alapú Motion Capture rendszer,” pp. 5, 7-9, 2011

[2] Mark S. Nixon, T. N. Tan, R. Chellappa, „Human Identification Based on Gait," New York, Springer Science + Business Media, Inc., 2006., p. 11.

[3] Ju Han, Bir Bhanu, „Individual Recognition Using Gate Energy Image," IEEE transactions on pattern analysis and machine intelligence, $\% 1$. szám28.2, pp. 316-322, 2006.

[4] Wei Zeng, Cong Wang, „Neurocomputing - View-invariant gait recognition via deterministic learning,",175, pp. 324-335, 2016.

[5] Salánki Dániel, Sarvajcz Kornél, Dr. Géza Husi: “Járásfelismerés fejlesztése NI LabVIEW környezetben", Debrecen, University of Debrecen, Mechatronical Engineering BSc, thesis, 2018.

[6] Salánki Dániel, „Járásfelismerés fejlesztése NI LabVIEW környezetben," University of Debrecen, Faculty of Engineering, National Council of Student Research Societies, Debrecen, 2017.

[7] Dacheng Tao, Xuelong Li, Xindong Wu, Stephen J. Maybank, „General Tensor Discriminant Analysis and Gabor Features for Gait Recognition," IEEE transactions on pattern analysis and machine intelligence, pp. 1-35, 2007.

[8] Khalid Bashir, Tao Xiang, Shaogang Gong, „Gait recognition without subject cooperation,” Pattern Recognition Letters, pp. 2052-2060, 2010.

[9] Chin Poo Lee, Alan W.C. Tan, Shing Chiang Tan, „Gait recognition with Transient Binary Patterns,” J. Vis. Commun. Image R., pp. 69-77, 2015.

[10] Gálai Bence, Benedek Csaba, „Járás alapú személyazonosítás és cselekvésfelismerés LiDAR szenzorokkal", 2017. 